v. 01, n. $01:$ pp. $57-66,2005$

ISSN $1808-0448$
Revista Gestão Industrial

\title{
EL SEDENTARISMO, NO ES SÓLO CAUSA DE LA TECNOLOGIZACIÓN
}

\section{THE SEDENTARISM IS NOT ONLY CAUSED BY THE TECHNOLOGICAL DEVELOPMENT}

\author{
Hugo Aránguiz Aburto ${ }^{1}$ \\ ${ }^{1}$ Departamento Educación Física, Universidad de Concepción, Chile, harangui@udec.cl
}

Recebido para publicação em: 03/12/2004
Aceito para publicação em: $25 / 03 / 2005$

\section{RESUMEN}

La relación de actividad física y salud, ha sido un eje estructurador de la Educación Física desde su constitución. Esta asociación positiva es muy antigua, y aparece claramente establecida en la cultura Griega. Existe, sin embargo, diferencias significativas en la representación de la actividad física que es considerada buena para la salud entre las culturas, en el transcurso del tiempo. Esas diferencias, se sitúan en el marco general de la comprensión acerca de una buena vida o sobre la vida que merece ser vivida, o calidad de vida que es un concepto multifactorial que implica tener salud física y mental, relaciones sociales, proteger e interactuar con el medio ambiente, educar para tener ciudadanos capaces para ejercer su civilidad, tiempo libre para disfrutar su ocio y cultura que trasciende las necesidades básicas de supervivencia. En la actualidad el valor de la autonomía física o corporal aparece como dimensión significativa de la salud y buena vida.

Palabras clave: Sedentarismo, actividad física

\section{Introducción}

En un recorrido histórico, en la segunda mitad del siglo XIX encontramos que el ejercicio físico fue orientado como instrumento de desarrollo y rendimiento motor humano, que permitía soportar los esfuerzo demandados por largas y pesadas jornadas de trabajo en las industrias. Así, el esfuerzo físico aparece como la solución para soportar la fatiga provocada por la explotación industrial. Esta misma orientación tiene la actividad física en las distintas guerras en que el hombre se ha visto enfrentado, que busca aumentar la resistencia al esfuerzo físico para movilizarse rápido, cruzar fronteras e invadir un país limítrofe o distante.

La metáfora del motor humano o máquina del rendimiento presenta variadas criticas de autores al asociarse con una base productivista, militarista y nacionalista, y algunas abiertamente racista y eugenésica de los movimientos contra el sedentarismo. Es el caso de Hitler que en su locura de la Raza superior prefería a las personas simples y fuerte a las personas débiles e 
intelectuales. En esta etapa el esfuerzo físico estuvo destinado a soportar el exceso de trabajo físico a través del entrenamiento del cuerpo.

En su evolución histórica, la jornada de trabajo tuvo una reducción significativa, sobretodo en las fábricas, así en el transcurso de un siglo la jornada de trabajo semanal declina aproximadamente a la mitad, y por otro lado el esfuerzo del trabajo humano fue substituido por motores movido por energía que no es del ser humano. De esta forma, el trabajo pesado que requiere gran esfuerzo y gasto energético, el cual realizaba gran parte de la población pasa a realizar trabajos más livianos, lo que trae consigo que los propósitos de incrementar las capacidades de fuerza y resistencia no se justifican en este nuevo contexto social.

Posteriormente, en el periodo conocido como guerra fría, en los años 45 al 90 del siglo XX aproximadamente, el conocimiento acerca de las ciencias del deporte logran un gran desarrollo que se transfiere y se aplica en la optimización del entrenamiento de los deportistas de competición. El deporte masivo, más allá de sus objetivos ideológicos es propuesto como la base de la cual se seleccionan los atletas o deportistas que aportaran medallas y será motivo de orgullo a sus países.

Paralelamente, producto de la intelectualización se genera un desarrollo en calidad y cantidad de la tecnologización que provoca la ruptura básica del equilibrio mente y cuerpo, por la progresiva inhibición de uno de ellos. No es sólo un producto de reciente sedentarismo, sino que tal profundo desequilibrio se origina con la intelectualización sectorial, que olvidada la acción, se cristaliza en una concepción educativa dualista mayoritariamente vigente hasta nuestro días. De la educación se derivó una valoración general del hombre brutalmente sectorizada: cuerpo y acción perdieron su significado humano. (CAGIGAL, J.M.,1986: 126)

¿Cuales son los cambios que se ha producido en el Concepto de Actividad Física en la actualidad?

En una forma sintetizada podemos considerar que la percepción anterior de la Actividad Física, ha tenido un cambio favorable que parte desde el área de la Salud y que ha sido asimilado en forma paulatina por el sector educacional, relacionando lo corporal, lo intelectual y lo espiritual en una perspectiva integradora, que se puede resumir en los siguientes aspectos :

- Antiguamente, la Actividad Física era necesaria para soportar la fatiga producto del esfuerzo laboral; Actualmente, se requiere actividad Física para compensar la disminución del esfuerzo físico en una sociedad tecnologizada;

- Los estímulos fisiológicos que se requieren para mantener una condición física se modifican desde los esfuerzos intensivos a los moderados. En este cambio, se pretende captar al ciudadano 
medio, que no esta ligado al deporte o percibe el deporte como un buen espectáculo de ser observado pero no practicado;

- $\quad$ Entre el deportista entrenado y el sedentario, surge la persona activa que requiere la actividad física para mantenerse saludable o entregar una imagen estéticamente optima como factor de marketing en una sociedad de consumo;

- La Actividad Física es considerada como un condicionante de Salud para combatir las enfermedades cardiovascular, obesidad, salud mental, tabaquismo y drogadicción ${ }^{1}$;

- En la generación de hábitos y conductas sociales requeridos por nuestra sociedad, también se recurre como la píldora o el antídoto adecuado para solucionar los males sociales como delincuencia y drogadicción que se padece actualmente.

\section{Causas que potencian el sedentarismo}

En un informe entregado por la O.M.S. ( Organización Mundial para Salud ), considera en forma estimativa que dos millones de muertes anuales pueden atribuirse a la inactividad física. Además, se presenta como una de las causas que aumenta las causas de mortalidad, duplica el riesgo de enfermedad cardiovascular, de diabetes de tipo II y de obesidad, aumenta el riesgo de padecer cáncer de colon y de pecho, hipertensión arterial, problemas de peso, osteoporosis, depresión y ansiedad.

Los datos obtenidos con las encuestas sobre salud realizadas en todo el mundo son notablemente similares, tanto en países desarrollados como en desarrollo. El porcentaje de adultos sedentarios o casi sedentarios se sitúa entre el $60 \%$ y el $85 \%$.

La OMS está actualmente evaluando el porcentaje mundial de enfermedades causadas por 22 factores de riesgo relativos a la salud, entre los que se encuentra la inactividad física. Esto hace necesario propiciar medidas de salud pública eficaces para promover la actividad física y mejorar la salud pública en todo el mundo.

Las causas o factores que potencian el Sedentarismo son de diverso orden que pueden estar referidos a sobre carga laboral, cambios tecnológicos; cambios de actitudes individuales sobre la actividad física, cambios en el medio ambiente; cambios en la comunicación; crecimiento urbano horizontal; menos áreas de recreación y de lugares para pasear ; inseguridad en las calles; adicción a TV e Internet; tecnología de lo confortable; hábitos alimenticios o regímenes alimenticios insanos; hacen de la actividad física una opción difícil.

\footnotetext{
1 "Se revaloriza a nivel internacional la influencia de los diferentes determinantes de la salud, en particular los de carácter socio-económico, psicosociales y ambientales para enfrentar los riesgos de enfermar. Primera Conferencia Mundial de Promoción de Salud en Ottawa en 1986, Directrices que continúan reforzándose por OPS/OMS y los Gobiernos en las posteriores Conferencias Mundiales, hasta llegar a la Quinta Conferencia Mundial de México 2000.”
} 
Para cada uno de estos factores se han entregado soluciones tendientes a crear un estilo de vida activo, así en los distintos programas de Actividad Física se han propiciado actividades en los lugares de trabajo- pausas activas- preferir el caminar para acercarse a los lugares de trabajo o de estudio, recrearse en grupos que favorezca la comunicación social, utilizar el elemento lúdico que permita una satisfacción y alegría por la actividad física, crear áreas de esparcimiento y recreación que permita un contacto con lo natural y sensibilizar acerca del cuidado de su medio ambiente, entrega de información de ejercicios y beneficios que presenta para la salud de ser activo. y campañas acerca de una buena alimentación para evitar una obesidad creciente.

Aún cuando son conocidos los múltiples beneficios que proporciona la actividad física para la salud, solamente una minoría de las personas realizan prácticas a un nivel que corresponda a la recomendación publicada en los Estados Unidos en el año 1993 que sugiere:

Cada norteamericano adulto debe consagrar a diario 30 minutos, al menos, a una actividad física de intensidad moderada. Se recomienda de igual manera la práctica regular de actividades que desarrollen y mantengan la fuerza muscular y la flexibilidad de las articulaciones. American College of Sports Medicine (A.C.SM.) 1993.

En todas las edades, desde la infancia a la vejez, tanto para el individuo que goza de buena salud como para el aquejado por una enfermedad crónica o una minusvalía se recomienda la máxima anteriormente expresada. Sin embargo, la proporción de individuos que practican en forma regular una actividad deportiva disminuye claramente a partir de los 19 a 20 años (VUORIi,I. y FENTEM P.1996: 15-17).

Conseguir aumentar en proporciones importantes la participación de las personas en los distintos grupos etáreos constituye una tarea apremiante. Para el éxito de esta empresa, existe consenso respecto a:

- $\quad$ movilización de medios considerables para sensibilizar a la opinión pública;

- crear condiciones para practicar actividades físicas sencillas para ser realizadas en las distintas edades;

- $\quad$ proporcionar servicios logísticos haciendo que parques, terrenos de juego, plazas, pistas y senderos sean también, en lo posible, accesibles al público;

- Cautelar muy especialmente para que se conceda un elevado grado de prioridad en los programas escolares, a la Educación Física y a la Educación para la Salud;

- Promover en las escuelas, la práctica regular de los deportes y de las actividades físicas en su tiempo libre. Los establecimientos educacionales ocupan una posición clave para suscitar entre los jóvenes la formación de hábitos de estilo de vida activa y saludable, de manera que lo conserven durante toda su vida. 


\section{3. ¿Cómo se ocupa el tiempo en la actualidad?}

Al respecto es importante, en la forma como es ocupado el tiempo por los niños como advierte el especialista Richard Strauss, director del Departamento de Control de Peso Infantil de la Escuela de Medicina de New Jersey:

Los tiempos en que los niños pasaban la mayor parte del día jugando al aire libre han quedado muy lejos. Hace todavía 20 años, en la televisión sólo se veían cinco canales y los chicos preferían estar afuera, jugando. Ahora la casa cuenta con más de 60 canales de televisión, computadoras y juegos de videos y el refrigerador está lleno de comida 'chatarra'.

En el adulto, el uso del tiempo también ha producido cambios que ha modificado su estilo de vida, producto de los distintos factores enunciado anteriormente lo que trae consigo la preocupación por el tiempo que se dispone para el disfrute personal . En el adulto como el niño, el tiempo es usado básicamente en cuatro grandes actividades : dormir, estudiar o trabajar, traslado y alimentación, y tiempo libre para disfrute personal.

Es indudable que en una cuantificación de un tiempo libre pasa por una consideración socioeconómica, que signifique disfrutar de más o menos tiempo, así , en una sociedad de consumo se estimula aumentar los ingresos para aumentar el poder comparador lo que lleva consigo una sobrecarga laboral.. Por otra parte, de acuerdo al estrato o nivel social, el uso de ese tiempo libre pasa por experiencias vividas de espectador (pasivo ) de espectáculos deportivos a una persona activa que ha vivenciado el proceso de comunicación corporal; las distancias a recorrer a sus lugares de trabajos o estudios es un aspecto importante en cuanto al tiempo real con el que se puede contar para su recreación, y por último los intereses particulares en cuanto a destinar más tiempo a lo laboral o estudio. Por lo tanto, una estimación del tiempo libre que cada persona puede tener para su desarrollo o goce personal es relativo, y que en una aproximación podemos estimar que puede fluctuar entre dos a cuatro horas de acuerdo a las consideraciones anteriores. En una estimación hipotética , una persona puede dividir su tiempo en:
a) dormir (8 horas)
b) estudiar o trabajar ( 8 horas)
c) tiempo libre : 4 horas
d) Traslado a lugares de trabajo y Alimentación : 4 horas 
Esto hace importante, crear condiciones y orientaciones que debemos entregar para el uso del tiempo libre, en forma beneficiosa a la salud del hombre como a los propósitos que busca la sociedad, acordes con las necesidades actuales determinados por políticas educacionales que se sustentan en cada país

\section{El sedentarismo en Chile}

Chile es un país altamente urbanizado, según el censo del 2002 , que nos entrega una distribución de 13.044.221 en el área urbana ( 86\% ) y 2.006.120 (14\%)en el área rural, con lo cual ha mejorado el acceso al agua potable, alcantarillado, educación y servicios de salud. Sin embargo, los niveles de calidad de vida presenta una tendencia negativa en los sectores más pobres, en aspectos como alimentación inadecuada, el sedentarismo, el consumo excesivo de tabaco, alcohol y drogas, la contaminación del aire y falta de espacios para recreación en las grandes ciudades, junto con el aumento de la delincuencia, violencia e inseguridad en las personas. El ingreso per cápita aumento en la década del 90, pero no disminuyó las desigualdades sociales, manteniendo un $20 \%$ de la población categorizado en un nivel de pobreza y con un $7 \%$ dela población en una categoría de extrema pobreza.

Población por áreas urbana y rural, resultados preliminares -Censo 2002

\begin{tabular}{ccccc}
\hline Total & Área Urbana & \% & Área Rural & $\%$ \\
\hline 15.050 .341 & 13.044 .221 & 86 & 2.006 .120 & 14 \\
\hline
\end{tabular}

Gran parte del aumento de ingreso, en especial en el sector más pobre, se destinó a comida rica en grasa y azúcar refinada, como es la comida chatarra y bebidas gaseosas, a compra de televisores, electrodomésticos y automóviles, con lo que disminuyó la actividad física y se incrementó el consumo de tabaco y alcohol, que inciden directamente en la salud de las personas. Esto significó un aumento explosivo de la obesidad y sedentarismo, así como del tabaquismo en la población chilena, especialmente en los niños, adolescentes y mujeres en edad fértil; fenómeno que también se ha ido produciendo en muchos países.(SALINAS J. y VIO F., 2002) . 
Tabla 1 - Uso de Tecnología y del tiempo según Fuente INE - 2002

\begin{tabular}{lll}
\hline \multicolumn{1}{c}{ Población Urbana } & \multicolumn{1}{c}{$\mathbf{8 6 \%}$} \\
\hline & 1970 & 2002 \\
Vehículos & 300.000 & 2.200 .000 \\
TV (a color) & 17.000 & 2.300 .000 \\
Horas frente a la TV & Días de la semana & $2-3$ hrs diarias \\
Horas de permanencia en el & Fines de semana & $4-5$ hrs diarias \\
trabajo & 50.6 hrs semanales (Región Metropolitana) \\
Horas de traslado al trabajo & $1-3$ hrs/dia \\
\hline
\end{tabular}

En la alimentación, las tendencias de consumo nacional entre el año 1980 y 2000, muestran un importante aumento en el consumo de carnes, en particular de cerdo y pollo, de cecinas, de productos lácteos y un bajo consumo de pescado, frutas, verduras, cereales y leguminosas.

En investigaciones respecto a la Actividad física en Chile, en dos encuestas sobre factores de riesgo de Enfermedades Crónicas No Transmisibles efectuadas en la Región Metropolitana de Santiago en 1988 y 1992 en población mayor de 15 años demostró que el 60\% de los hombres y el $80 \%$ de las mujeres hacían menos de 15 minutos de actividad física continua dos veces por semana (BERRIOS, X,1997).

En la Encuesta Carmen( JADUE L. y OTROS) aplicada en 1997 en Valparaíso a una muestra representativa de población mayor de 25 años demostró que el $93 \%$ de las mujeres no realizaban ningún tipo de actividad física en su tiempo libre, lo cual subía al $97 \%$ en el nivel socioeconómico bajo.

Figura 1 - Sedentarismo 


\section{SEDENTAR ISMO}

Prevalencia de Sedentarismo según sexo

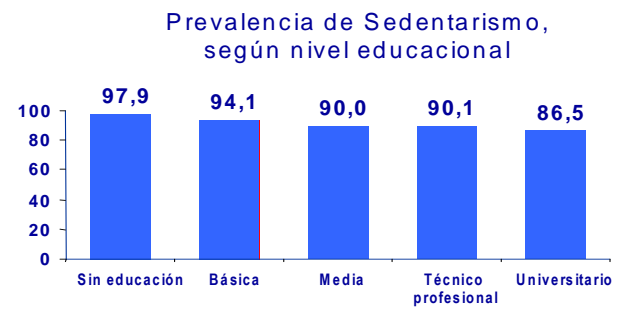

Fuente: Encuesta de Calidad de Vida INE/MINSAL2000

Existen dos encuestas sobre actividad física efectuadas el año 2000 con la misma metodología y resultados similares: la Encuesta de Caracterización Socioeconómica (CASEN) efectuada en mayo en 20.000 hogares y la Encuesta Nacional de Calidad de Vida y Salud 2000 (ECVS) en 6.000 hogares, con representatividad a nivel regional y urbano-rural, efectuada en diciembre.

La pregunta en ambas encuestas fue ¿Cuántas veces ha practicado deporte o alguna actividad física (en sesiones de no menos de 30 minutos), dándose como alternativas posibles: 3 o más veces por semana; 1 a 2 veces por semana; menos de 1 vez por semana y ninguna. El porcentaje de población que no hace ninguna actividad física es de un 71\% (CASEN) y 73\% (ECVS) y los que hacen mas de 30 minutos tres veces por semana fue de 9,4\% y $9 \%$ respectivamente. En ambas encuestas el sedentarismo es mayor en las mujeres y en los niveles socioeconómico bajos.

Figura 2 - Porcentaje de población que hace actividad física

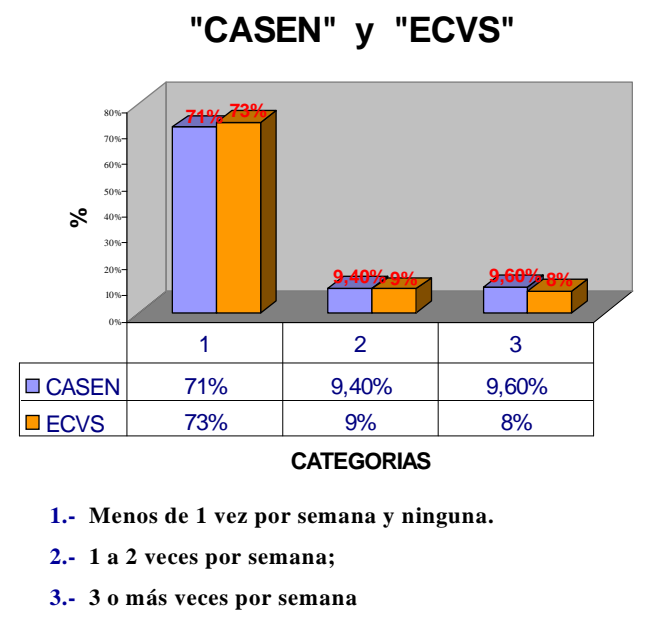


La relación de tabaquismo con la Actividad Física ha sido relacionada con la disminución de los niveles de capacidad aeróbica por ser el humo de tabaco el contaminante más importante en los espacios interiores.. El tabaquismo muestra un aumento en Chile en el año 2000, dos estudios nacionales coincidieron en que el 40\% de la población mayor de 12 años (CONACE)) y de 15 años (ECVS) dice haber consumido tabaco en el mes anterior. Por otra parte, esta conducta es más frecuente en hombres que en mujeres y en el sector urbano es mayor que en el rural.

Los determinantes psicosociales, tanto internos o propios del individuo-autoestima, afectividad, estrés, satisfacción vital- como los externos-relaciones interpersonales y sociales- , en la ECVS muestra que el 35\% de la población se declara con estrés y que el $46 \%$ pertenece a alguna organización social, siendo las mas frecuentes las religiosas, deportivas, centros de padres y apoderados y de adultos mayores.

Finalmente, otro aspecto relacionado con el sedentarismo son los factores ambientales que en la ECVS indica que lo que más preocupa a la población son la falta de espacios públicos para la recreación y el deporte (plazas, áreas verdes y equipamiento comunitario) junto con problemas de contaminación e higiene. En los problemas de contaminación y seguridad pública son mayores en los sectores urbanos, y en lo rural las principales preocupaciones son los problemas de aislamiento y falta de infraestructura comunitaria (SALINAS J.; VIO F., 2002).

\begin{abstract}
The relation between physical activity and health has been a guider axle of Physical Education since its creation. This positive association is very old, and appears clearly established in Greek culture. There are, undoubtedly, significant differences in the physical representation that is considered for health among the cultures, and in the time course. These differences, are located in the general frontier of the understanding of a good quality of life or of the life that deserves to be lived, or quality of life, that is a multifactorial concept, which implies in having physical and mental health, social relations, protecting and interacting with the environment, educating to have capable citizens to exert their civility, free time to enjoy their idleness and culture that exceeds the basic necessities of survival. Currently, the value of the physical or corporal autonomy appears as a significant dimension of health and good quality of life.
\end{abstract}

Keywords: Sedentarism, physical activity and health, physical education

\title{
5. Referências
}

ARANGUIZ, H. Enfoques de la actividad física orientados hacia la obtención de una mejor salud. In: CONGRESO CIENTIFICO LATINOAMERICANO DA FIEP, 2, 2002, Piracicaba. Anais... Piracicaba : Unimep, 2002.

BELLO, S.; SOTO, M.; SALINAS, J. Salud sin tabaco. Guía técnica-metodológica. Programa Ambientes Libres del Humo de Tabaco. Impresos La Nación SA. Ministerio de Salud, 2001.

BERRÍOS, X.; JADUE, L.; CENTENO, J.; ROSS, M. I.; RODRÍGUEZ, H. Prevalencia de factores de riesgo de enfermedades crónicas: un estudio poblacional en la Región Metropolitana de Santiago, Chile. 1986-1987. Rev. Med. Chile, n. 118, p. 597-604, 1990. 
BERRÍOS, X. Factores de riesgo en enfermedades crónicas de adultos. Un ejemplo de investigación epidemiológica. Boletín Esc. Medicina P. Universidad Católica de Chile, n. 23, p. 73-89, 1994.

CAGIGAL, J. M. ¡Oh deporte! (anatomía de un gigante). España : Editorial Miñon, 1981.

CRUZ, E. El tabaquismo en el nivel primario. Vida Médica Santiago, Chile, n. 43, p. 44-46, 1991.

INSTITUTO NACIONAL DE ESTADÍSTICAS. Censo 2002. Chile : INE, 2002.

JADUE, L.; VEGA, J.; ESCOMAR, M. C.; DELGADO, I.; GARRIDO, C.; LASTRA, P.; ESPEJO, F.; PERUGA, A. Factores de riesgo para enfermedades crónicas no transmisibles: métodos y resultados de la encuesta Carmen. Rev. Med. Chile, n. 127, p. 1004-1013, 1999.

JOLY, D. J. Encuesta sobre las características del hábito de fumar en América Latina. Washington, DC : OPS, 1997. (Publicación Científica; 337).

LOVISOLO, H. Atividade fisica e saúde: uma agenda sociológica de pesquisa. In: MOREIRA, W. W.; SIMOES, R. Esporte como fator de qualidade de vida. [S. 1.] : UNIMEP, 2003.

MINISTERIO DE PLANIFICACIÓN Y COOPERACIÓN, INSTITUTO NACIONAL DEL DEPORTE. Encuesta de Caracterización Socioeconómica Nacional (CASEN) 2000. Santiago : Sector Deportes, 2001.

MINISTERIO DE SALUD. Departamento de Epidemiología. Departamento de Promoción de Salud. Encuesta de Calidad de Vida y Salud. Chile, 2000.

SAliNAS, J.; VIO, F. Promoción de la salud en Chile. Rev. Chil. Nutr., v. 29, supl. 1, oct. 2002.

SANTIN, S. Qualidade de vida e esporte nos caminhos da filosofia da corporeidade. In: MOREIRA, W. W.; SIMOES, R. Esporte como fator de qualidade de vida. [S. 1.] : UNIMEP, 2003.

ViO, F.; AlBAlA, C.; CROVETTO, M. Promoción de salud en la transición epidemiológica de Chile. Rev. Chil. Nutr., v. 27, p. 21-29, 2000.

VIO, F.; ALBALA, C. Nutrition policy in the chilean transition. Public Health Nutrition, n. 3, p. 49-55, 2000.

VUORI, I.; FENTEM, P.; SVOBODA, B.; PATRIKSSON, G.; ANDREFF, W.; WEBER, W. La función del deporte en la sociedad. España : Ministerio de Educación y Cultura, 1996.

WELK, G. Physical activity assessments for health-related research. USA : Human Kinetics, 2002. 